\title{
Modified DV-Hop Algorithm for Localization in Wireless Sensor Networks
}

\author{
Glory Pachnanda \\ M. J. P. Rohilkhand University, Bareilly, U.P. India \\ e-mail: Glory.pachnanda@gmail.com
}

\begin{abstract}
Wireless sensor networks (WSNs) help monitoring physical environments with improved accuracy. Localization has been a major challenge in such networks because without finding the location of the sensor that is informing sensed data, the informed data will not be useful. Localization algorithm is categorized as: anchor based approach and anchor free approach in which DV-Hop algorithm is anchor based approach. In this paper, we modify the DV-Hop algorithm; deprivation of DV-Hop algorithm is that this algorithm works badly in sparse topology, so we have done some improvements shown in this paper and compare the simulation results of both algorithms (DV-Hop and Modified DV-Hop algorithm). The result shows that accuracy of modified DV-Hop algorithm is better than DV-Hop algorithm and modified DV-Hop algorithm also works in sparse networks.
\end{abstract}

Keywords: Anchor based, Anchor free, DV-Hop, MDVH, WSNs

\section{Introduction}

WSNs consist of small self governing nodes. Each node has a small microprocessor, a radio chip, some transducer and is usually battery powered which sets lifetime of network. Wireless sensor nodes monitor physical and environmental activity such as temperature, humidity, moisture, fire detection etc [1]. There are many challenges and open research questions in the area of WSNs. Some challenges are synchronization, coverage, security, energy consumption and localization.

Localization is the process in which location of sensor nodes is to be determined? In WSNs, sensor nodes are deployed in two manners: manually and randomly; in manual placement, the nodes are placed by human hand, it is difficult to deploy the nodes manually in inhabitants area, so, random placement technique is used. In this technique, location of sensor nodes is not determine because nodes are thrown through the airplane or aircraft, so, information transferred by the nodes become useless for us. Thus, localization process is used to determine the location of sensor nodes. Localization algorithm is categorize as: anchor based and anchor free technique. In anchor based technique, the exact coordinates of a sensor node from a set of geometric constraints extracted from proximity measurements [2]. However, accuracy of the estimated positions is highly affected by the number of anchor nodes and their distribution in the sensor field [3]. In anchor free technique, the relative location of sensor nodes from a set of geometric constraints extracted from proximity measurements.

\section{DV-HOP Algorithm}

DV-Hop algorithm is summarized in figure 1. It consists of four phases, in the first phase, an anchor i floods a message containing its coordinates ( $\mathrm{x}, \mathrm{y})$ and a variable Hop Count ${ }_{i}$ that signifies the number of links (hops or connections) between the sensor that received this variable and the sending anchor i. Sensors store and exchange hop counters of anchor node. In the second phase, anchor I computes the hop length from its perspective $\mathrm{Hop}_{i}$, using equation (1) which is given as,

$$
\operatorname{Hop}_{i}=\frac{\sum_{j=1, i \neq j}^{M} \sqrt{\left(x_{i}-x_{j}\right)^{2}+\left(y_{i}-y_{j}\right)^{2}}}{\sum_{j=1, i \neq j}^{M} \text { HopCount }_{i, j}}
$$


Where, $\mathrm{M}$ is the number of anchors in the network, $\mathrm{j}$ identifies other anchor nodes Hop Count $_{i, j}$ is the distance in hops between anchor $\mathrm{i}$ and anchor $\mathrm{j},\left(x_{i}, x_{j}\right)$ and $\left(y_{i}, y_{j}\right)$ represent the coordinates of anchors $i$ and $j$. Where, $i=1,2,3,4 \ldots \ldots \ldots$ and $j=1,2,3,4 \ldots \ldots \ldots \ldots$.

After computing average hop length, anchor $i$ floods it in the network for other anchors and sensors. An unknown sensor node maintains only the average hop length flooded by the closest beacon to it. Unknown node uses the received average hop length to compute the distance $d_{j}$ between unknown node and anchor j using equation (2),

$$
d_{j}=\text { Hop }_{i} \times \text { HopCount }_{j}
$$

Where, HopCount ${ }_{j}$ is the hop counter that unknown node maintains for anchor $\mathrm{j}$.

In the third phase, a sensor uses the least square (LS) technique to trilaterate its position. The objective in this LS problem is to minimize the summation of the square of errors between the measured distances from the reporting sensor to each anchor obtained according to equation (2) and the distance between the relative anchor and the estimated position of sensor [4].

In the fourth phase, the localization error and accuracy can be calculated as,

$$
\left.e_{i}=\sqrt{\left(x_{i}\right.}-x_{j}\right)^{2}+\left(y_{i}-y_{j}\right)^{2}
$$

Where, $e_{i}$ is the localization error, $\left(x_{i}, y_{i}\right)$ and $\left(x_{j}, y_{j}\right)$ are the coordinates of anchor nodes and unknown node, and accuracy can be calculated as,

$$
\text { accuracy }=\frac{e_{i}}{R}
$$

Where, $\mathrm{R}$ is the communication range.

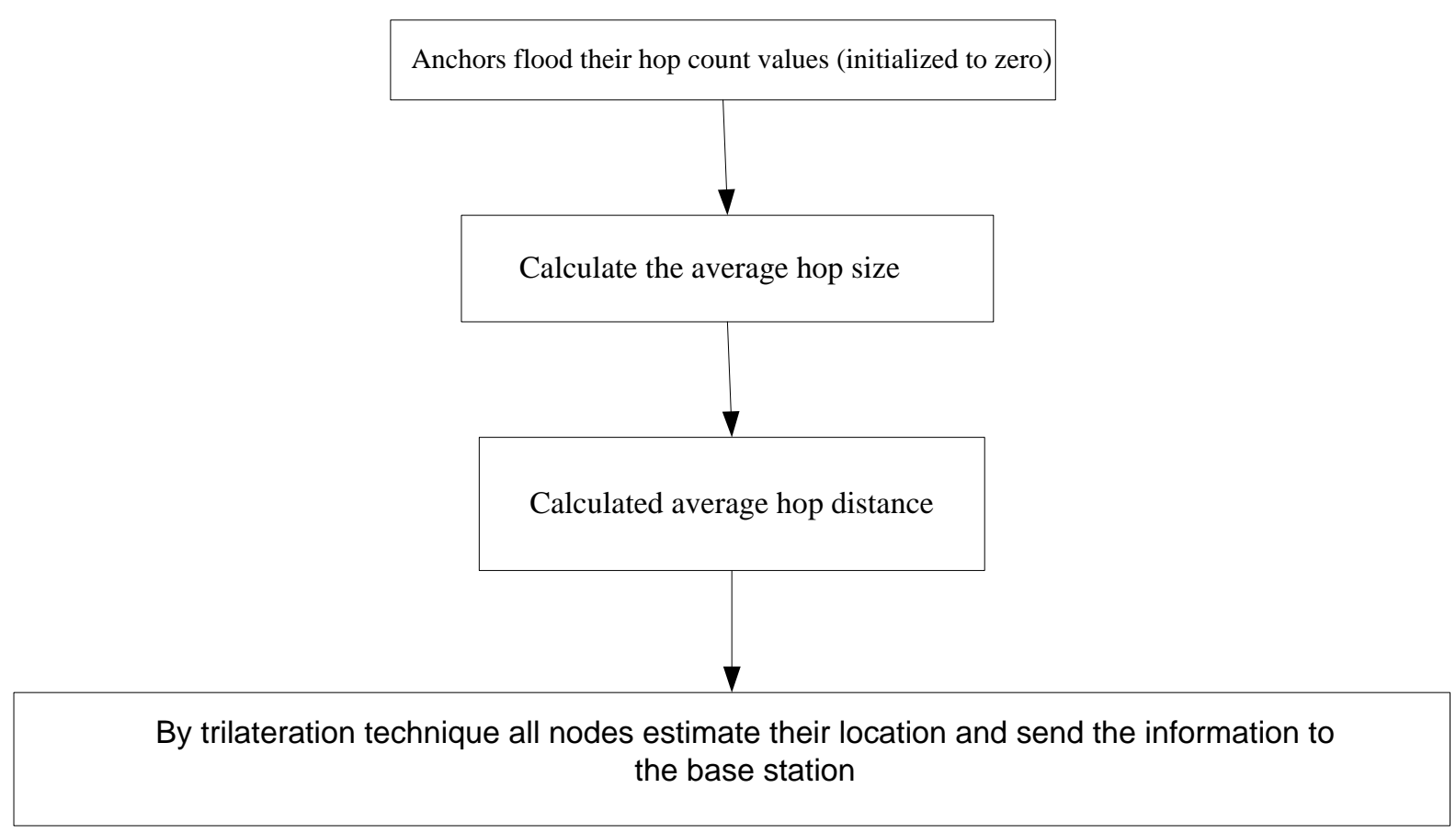

Figure 1. Flow chart of DV-Hop algorithm [5]

\section{Modified DV-HOP Algorithm}

Apart from above discussed existing improved DV-Hop algorithm, we propose a new algorithm for improving localization of sensor nodes. The proposed algorithm is based on the 
assumptions of DV-Hop algorithm and improves localization accuracy by reducing the localization error. The DV-Hop does not provide the accurate results if used in the sparse topology, but proposed algorithm can also be used in WSNs deployed in sparse topology.

In DV-Hop algorithm, the distance between anchor nodes and unknown nodes are used as the average hop distance. This algorithm has poor accuracy and works badly in sparse or irregular networks. Many researchers modify the disadvantage of DV-Hop localization algorithm. The key to reduce the localization error of DV-Hop algorithm is to improve accuracy of the distance calculation. The following assumptions are taken into account and are described in following steps:

\section{STEP I: INFORMATION BROADCASTING}

Each anchor node will convey the location information to neighboring nodes with hop count initialized to one. Broadcasting information format is $\left(i d_{i}, x_{i} y_{i}, H o p s_{i}\right)$, where, $i d_{i}$ and $\left(x_{i}, y_{i}\right)$ is identity number and coordinates of sensor node $\mathrm{i}$. each receiving node maintains the anchor node information and minimum hop count value per anchor of all nodes it receives.

If a node receives a packet with the same id, then it compares the existing hop value and new hop count value. If the new hop count is less than the hop already existing in the table, the new hop will update the information of hop in the table otherwise the packet will be discarded and no longer be forwarded. Each receiving node increases the hop count by one before transmitting it to other neighboring nodes.

\section{STEP II: DISTANCE CALCULATION}

Distance between the unknown node and the anchor node is estimate. The anchor node gets the hop values to other anchor node it estimate the hop size,

$$
\text { Hop }_{i}=\frac{\sum_{j=1, i \neq j}^{M} \sqrt{\left(x_{i}-x_{j}\right)^{2}+\left(y_{i}-y_{j}\right)^{2}}}{\sum_{j=1, i \neq j}^{M} \text { HopCount }_{i, j}}
$$

Where, $\mathrm{M}$ is the number of anchors in the network, HopCount ${ }_{i, j}$ is number of hops between anchor node $\mathrm{i}$ and $\mathrm{j} ;\left(x_{i}, y_{i}\right)$ and $\left(x_{j}, y_{j}\right)$ are coordinates of anchor node $\mathrm{i}$ and $\mathrm{j}$.

The average hop size calculated as,

$$
\text { HopSize }_{\text {avg }}=\frac{\sum H o p_{i}}{M}
$$

Distance $d_{j}$ between unknown node and anchor node is calculate by multiplying hop size by its minimum hop count and save it into the table,

$$
d_{j}=\text { HopSize }_{\text {avg }} \times \text { HopCount }_{i, j}
$$

\section{STEP III: LOCATION CALCULATION}

According to the distance information of many anchor nodes that has been obtained the unknown node, the unknown node calculates its coordinates using trilateration or multilateration measurement, 


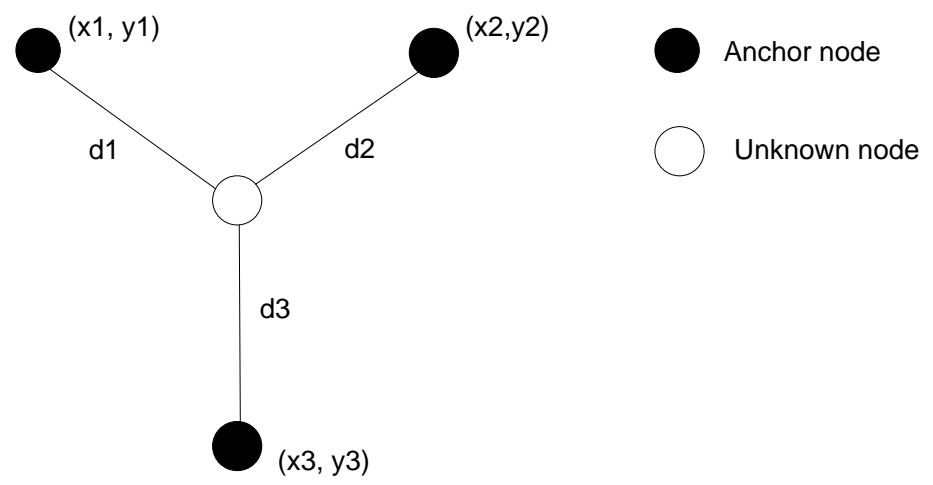

Figure 2. Location representation of unknown node

Using the distance formula,

$$
\begin{aligned}
& \left(x_{1}-x_{u}\right)^{2}+\left(y_{1}-y_{u}\right)^{2}=d_{1}^{2} \\
& \left(x_{2}-x_{u}\right)^{2}+\left(y_{2}-y_{u}\right)^{2}=d_{2}^{2} \\
& \left(x_{3}-x_{u}\right)^{2}+\left(y_{3}-y_{u}\right)^{2}=d_{3}^{2}
\end{aligned}
$$

Subtracting equation (10) from (8) and (9),

$$
\begin{aligned}
& \left(x_{1}-x_{u}\right)^{2}-\left(x_{2}-x_{u}\right)^{2}+\left(y_{1}-y_{u}\right)^{2}-\left(y_{2}-y_{u}\right)^{2}=d_{1}^{2}-d_{2}^{2} \\
& \left(x_{2}-x_{u}\right)^{2}-\left(x_{3}-x_{u}\right)^{2}+\left(y_{2}-y_{u}\right)^{2}-\left(y_{3}-y_{u}\right)^{2}=d_{2}^{2}-d_{3}^{2}
\end{aligned}
$$

From equation (11) and (12),

$$
\begin{aligned}
& 2\left(x_{3}-x_{1}\right) x_{u}+2\left(y_{3}-y_{1}\right) y_{u}=d_{1}^{2}-d_{3}^{2}-x_{1}^{2}+x_{3}^{2}-y_{1}^{2}+y_{3}^{2} \\
& 2\left(x_{3}-x_{2}\right) x_{u}+2\left(y_{3}-y_{2}\right) y_{u}=d_{2}^{2}-d_{3}^{2}-x_{2}^{2}+x_{3}^{2}-y_{2}^{2}+y_{3}^{2}
\end{aligned}
$$

From equation (13) and (14),

$$
2\left[\begin{array}{ll}
\left(x_{3}-x_{1}\right) & \left(y_{3}-y_{1}\right) \\
\left(x_{3}-x_{2}\right) & \left(y_{3}-y_{2}\right)
\end{array}\right]\left[\begin{array}{l}
a_{u} \\
b_{u}
\end{array}\right]=\left[\begin{array}{l}
\left(d_{1}^{2}-d_{3}^{2}-x_{1}^{2}+x_{3}^{2}-y_{1}^{2}+y_{3}^{2}\right) \\
\left(d_{2}^{2}-d_{3}^{2}-x_{2}^{2}+x_{3}^{2}-y_{2}^{2}+y_{3}^{2}\right)
\end{array}\right]
$$

Equation (15) can be written as,

$$
\begin{aligned}
& \mathrm{A}=2\left[\begin{array}{ll}
\left(x_{3}-x_{1}\right) & \left(y_{3}-y_{1}\right) \\
\left(x_{3}-x_{2}\right) & \left(y_{3}-y_{2}\right)
\end{array}\right] \\
& \mathrm{B}=\left[\begin{array}{l}
\left(d_{1}^{2}-d_{3}^{2}-x_{1}^{2}+x_{3}^{2}-y_{1}^{2}+y_{3}^{2}\right) \\
\left(d_{2}^{2}-d_{3}^{2}-x_{2}^{2}+x_{3}^{2}-y_{2}^{2}+y_{3}^{2}\right)
\end{array}\right] \\
& \mathrm{X}=\left[\begin{array}{l}
a_{u} \\
b_{u}
\end{array}\right]
\end{aligned}
$$

From above equation, the coordinates of location node $U$ can be obtained using estimation methods of the least square method,

$$
\begin{aligned}
& X=A^{-1} B \\
& X=\left(A^{\top} A\right)^{-1} A^{\top} B
\end{aligned}
$$




\section{STEP 4: ESTIMATION OF LOCALIZATION ERROR AND ACCURACY}

The localization system is divided into three elements, distance estimation, position computation and the localization algorithm. Each element can affect on the error of the system. The influence of these errors in geographic algorithm is also analyzed, showing the importance of understanding the error behavior and the importance of geographic algorithms which consider the inaccuracy of location estimations. In DV-Hop algorithm, the location error and accuracy can be calculated as,

$$
\begin{aligned}
& \left.e_{i}=\sqrt{\left(x_{i}\right.}-x_{j}\right)^{2}+\left(y_{i}-y_{j}\right)^{2} / \mathrm{R} \\
& \text { Accuracy }=\frac{M}{e_{i}}
\end{aligned}
$$

\section{Simulation Results}

In this section we will present a framework for testing the performance of localization algorithms (DV-Hop and Improved DV-Hop algorithm). It provides the facility to researchers to check functioning of algorithm in different scenarios. The aim of this section is to determine the performance of the proposed algorithm. Algorithms are implemented in MATLAB.

Table 1. Simulation parameters

\begin{tabular}{cc}
\hline Parameters & Value \\
\hline Area & $100 \times 100 \mathrm{~m}^{2}$ \\
Communication range & $50 \mathrm{~m}$ \\
Sensor nodes & 250 \\
Anchor nodes & 50 \\
Unknown nodes & 200 \\
\hline
\end{tabular}

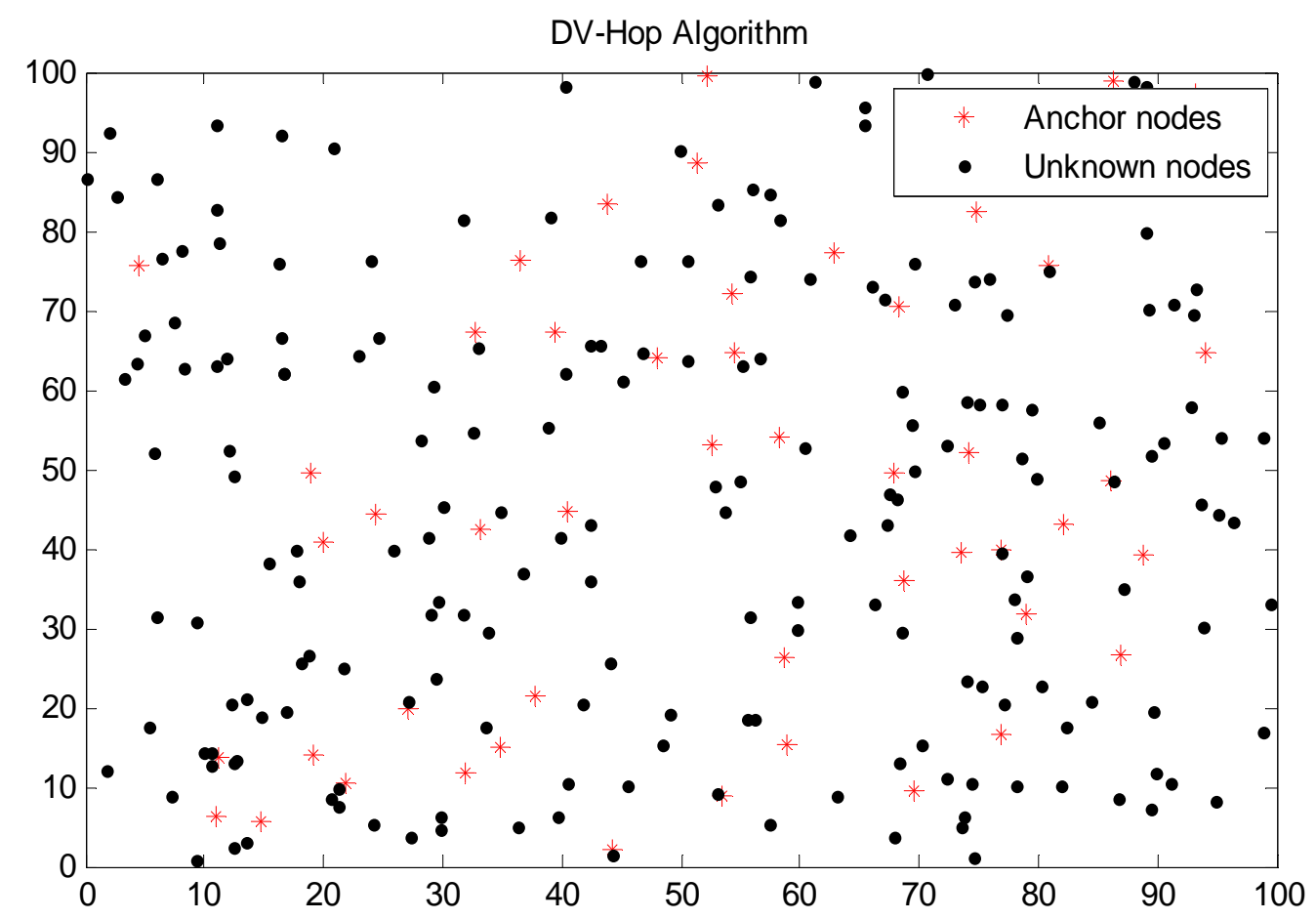

Figure 3. Random distribution of sensor nodes 


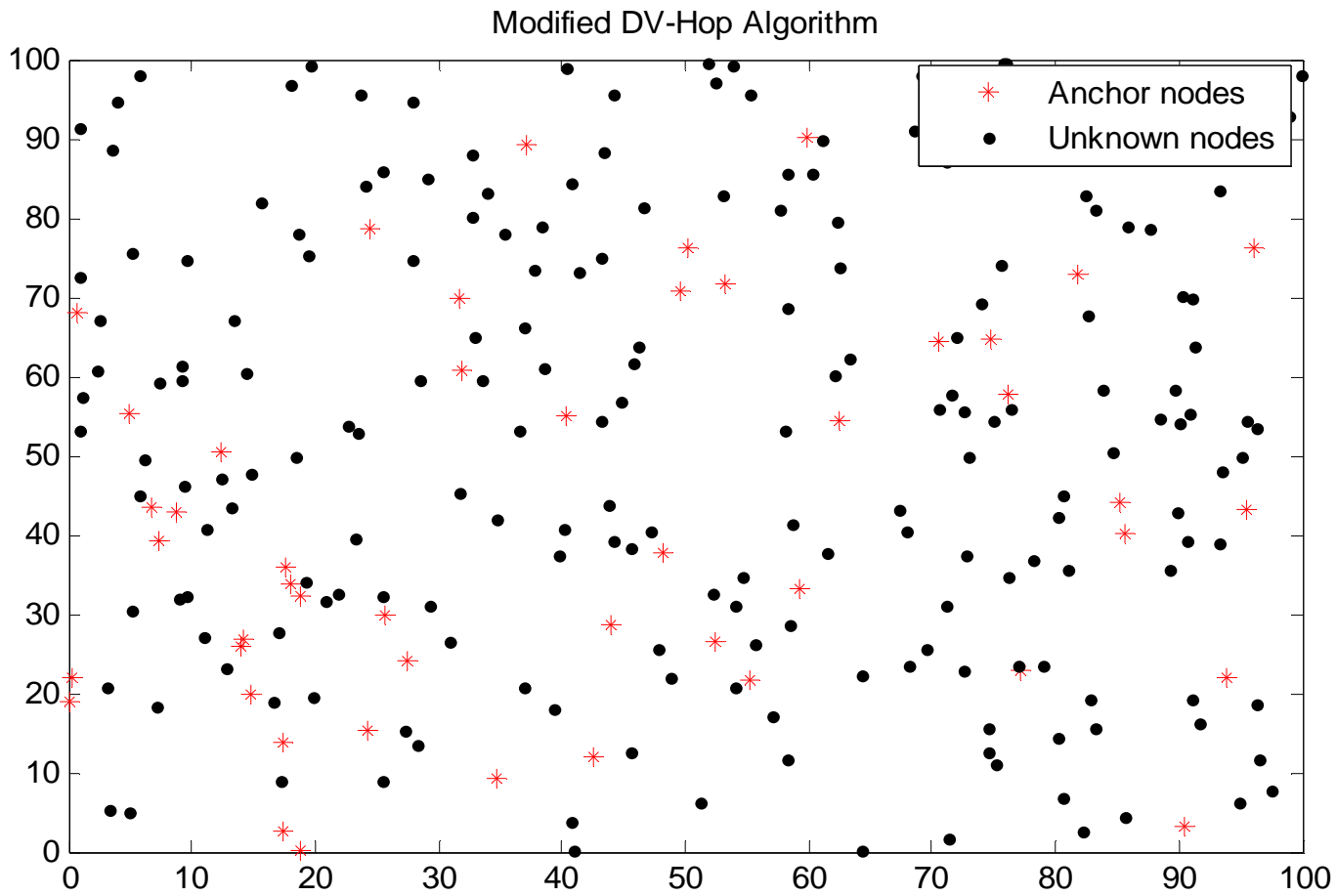

Figure 4. Random distribution of sensor nodes

Figure 3 and 4 shows the random distribution of 250 sensor nodes in the area $100 \times 100$ $\mathrm{m}^{2}$ in which 50 nodes are anchor nodes.

\subsection{Varying Number of Anchor Nodes}

Suppose that anchor nodes and unknown nodes are randomly distribute in a $100 \times 100$ $\mathrm{m}^{2}$ area in which the number of anchor node and the unknown node is 250 , communication range $\mathrm{R}=50 \mathrm{~m}$, when anchor node is respectively 10, 20, 30, 40, 50, 60, 70, 80, 90, 100. Simulation is done on two algorithms (DV-Hop algorithm and Modified DV-Hop algorithm). Figure 5 and Figure 6 shows the localization error and accuracy between DV-Hop and Modified DV-Hop algorithm, when varying anchor nodes. As the number of anchor nodes are increases localization error is reduces because more nodes know about their location, less nodes are left to estimate location. Anchor nodes help unknown node to identify its own location, so, accuracy is increases. 


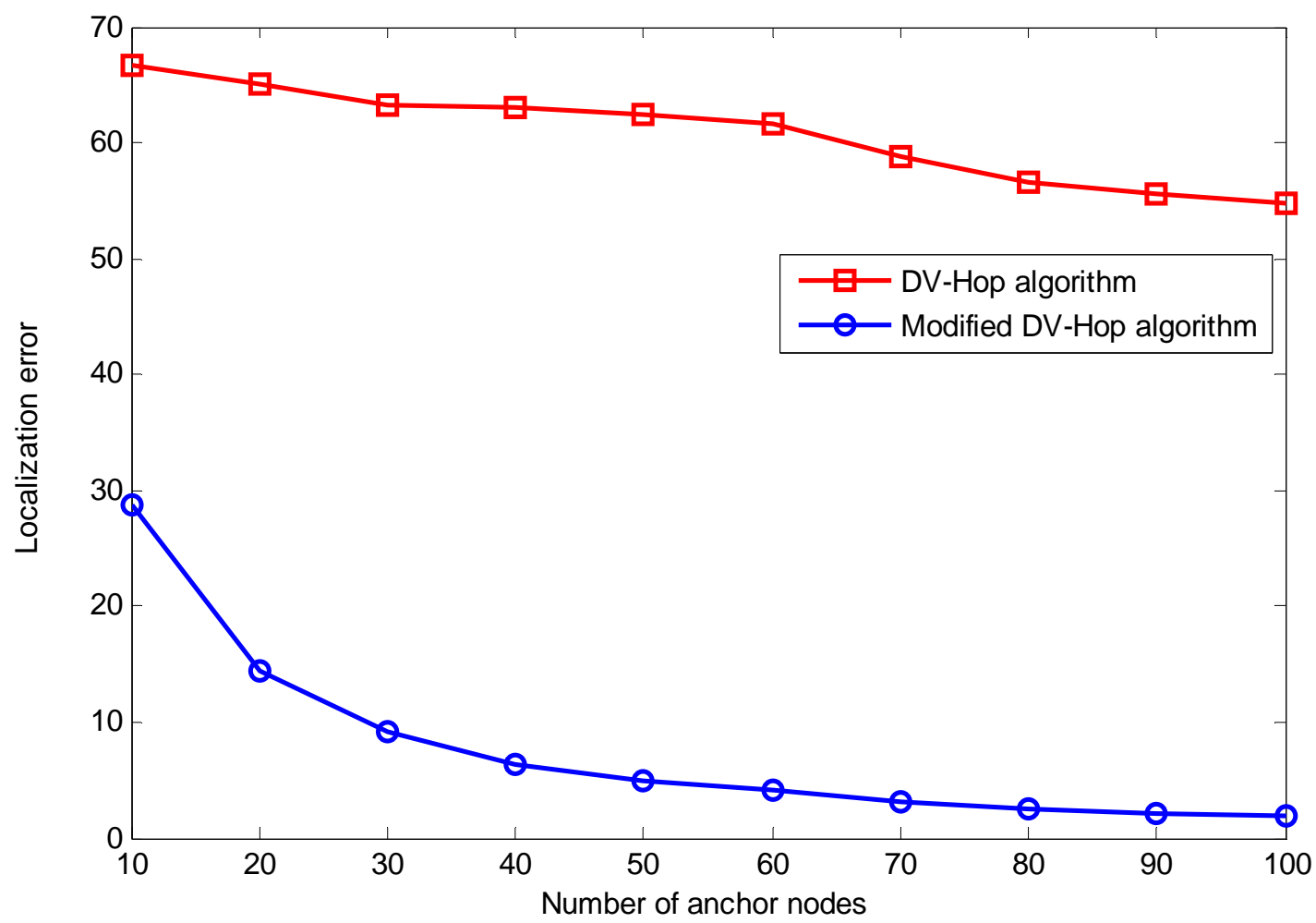

Figure 5. Localization error with number of anchor nodes

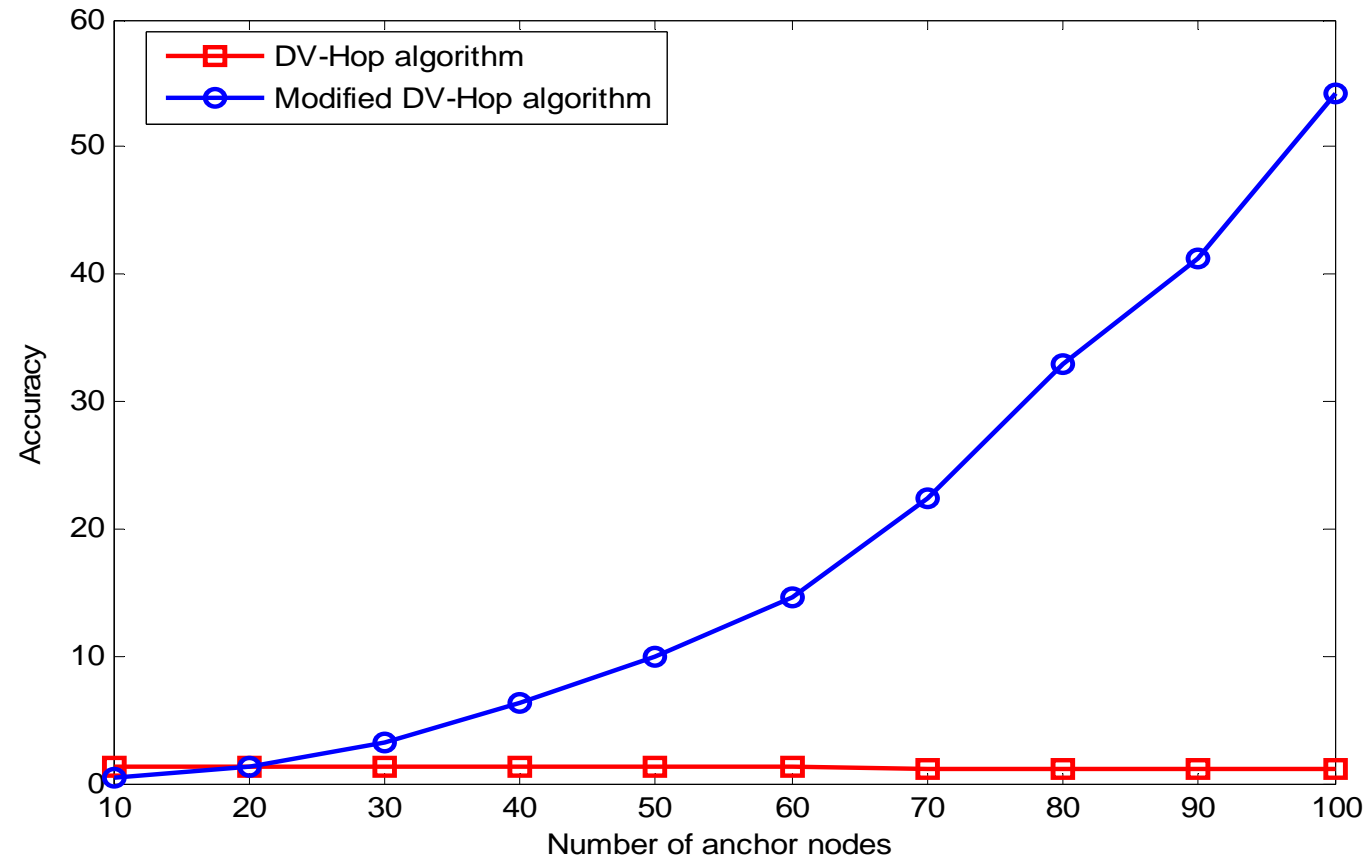

Figure 6. Accuracy with number of anchor nodes 


\subsection{Varying Communication Range}

Anchor nodes and unknown nodes are randomly distributed in a $100 \times 100 \mathrm{~m}^{2}$ area in which total amount of nodes is 250 . The node communication range respectively, $10 \mathrm{~m}, 20 \mathrm{~m}$, $30 \mathrm{~m}, 40 \mathrm{~m}, 50 \mathrm{~m}, 60 \mathrm{~m}, 70 \mathrm{~m}, 80 \mathrm{~m}, 90 \mathrm{~m}, 100 \mathrm{~m}$. Performance of both algorithms has shown in figure 7 and 8 . As the communication range increases, area covered by nodes is also increases. If communication range is increases than it cover more nodes. For example: anchor node know about their location and if the communication range of node is $100 \mathrm{~m}$ that means it cover $100 \mathrm{~m}$ area and the nodes which coming in $100 \mathrm{~m}$ range are able to identify their location.

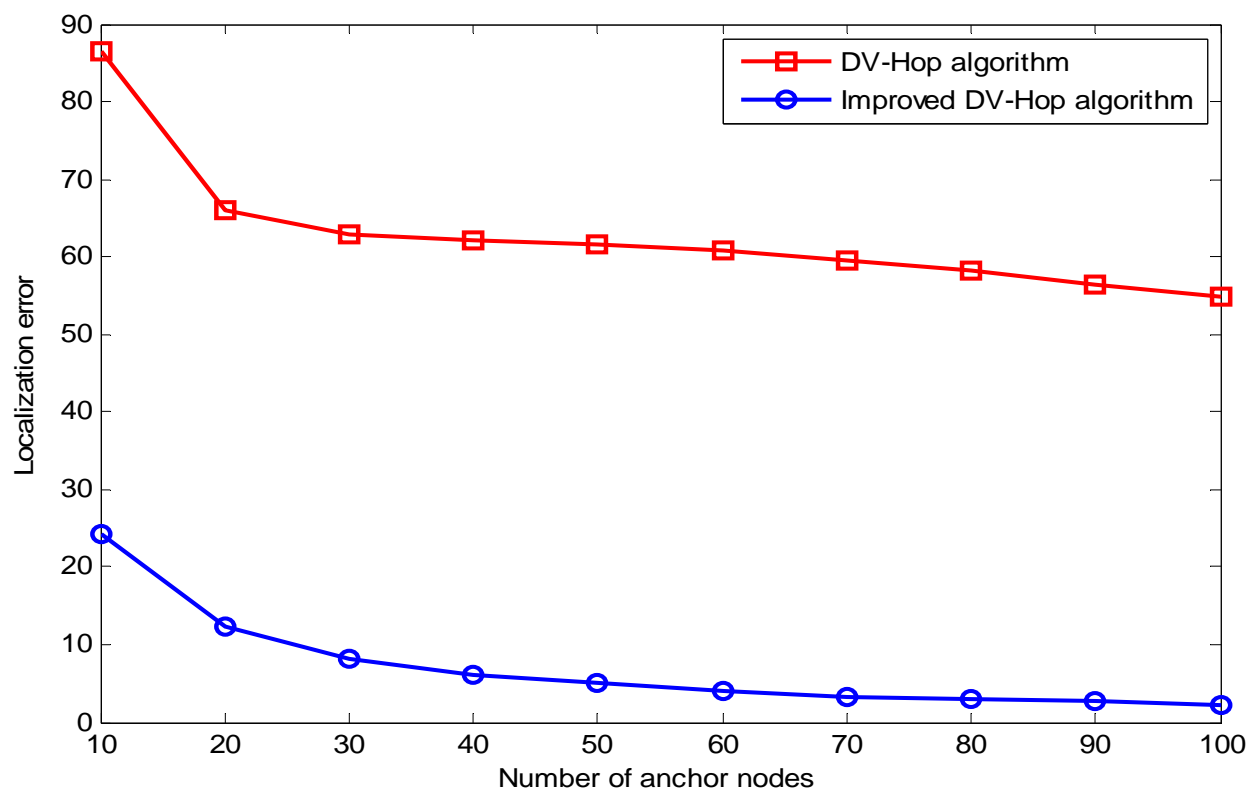

Figure 7. Localization error with communication range

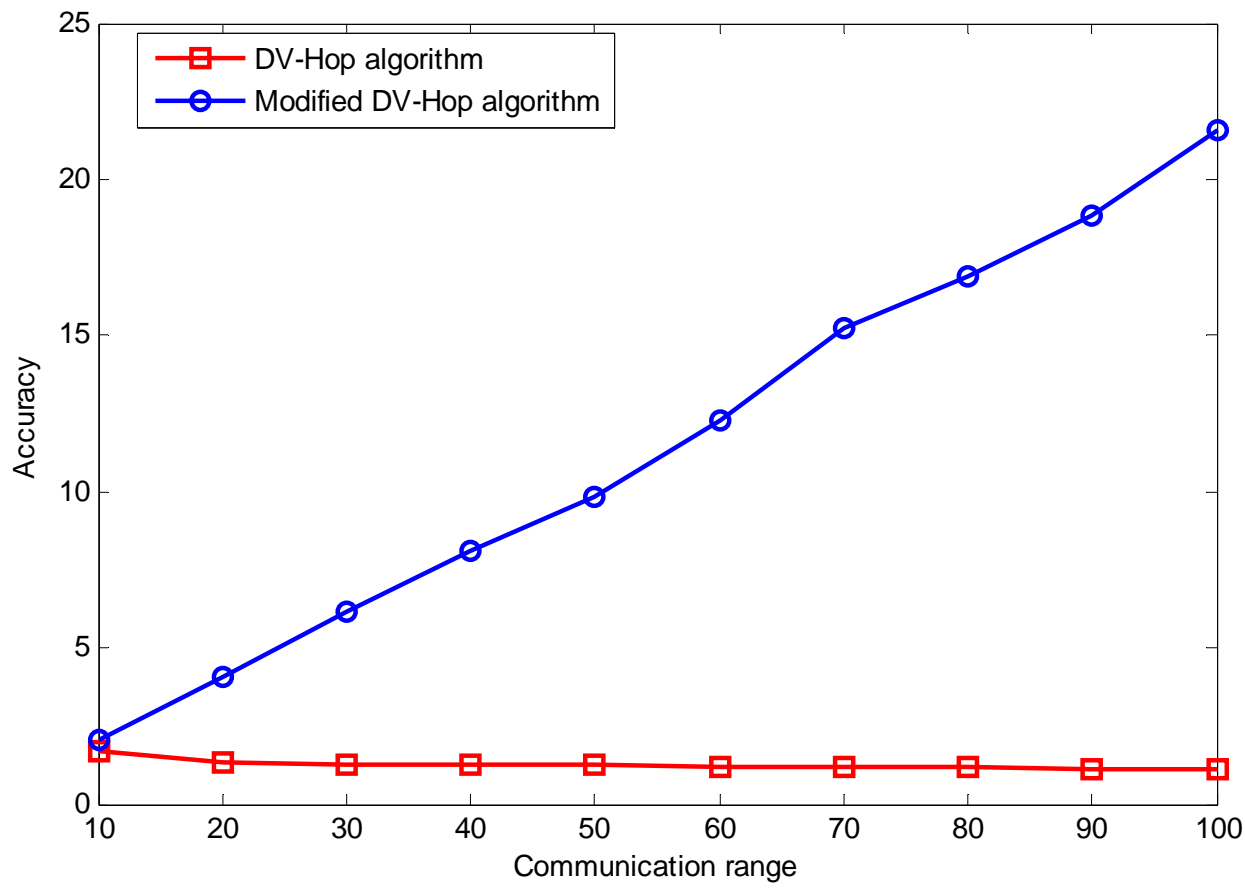

Figure 8. Accuracy with communication range 


\section{Comparative Results}

Table 2 summarizes the comparative results of both algorithms and shows that the modified DV-Hop algorithms has better accuracy and less simulation time and it reduces the localization error more than the DV-Hop algorithm.

Table 2. Comparative results of DV-Hop algorithm and modified DV-Hop algorithm Simulation Parameters: Area $=100 \mathrm{~m}^{2}$, Number of nodes $=\mathbf{2 5 0}$, Unknown nodes $=\mathbf{2 0 0}$, Anchor nodes $=\mathbf{5 0}$,

\begin{tabular}{|c|c|c|c|}
\hline \multicolumn{4}{|c|}{ Communication range $=50 \mathrm{~m}$} \\
\hline Algorithm & & Accuracy & Simulation time \\
\hline DV-Hop Algorithm & 59.6783 & 1.1936 & $10.382 \mathrm{sec}$ \\
\hline Modified DV-Hop Algorithm & 5.0376 & 9.9253 & $7.157 \mathrm{sec}$ \\
\hline
\end{tabular}

This section comprises the existing improved DV-Hop and the Modified DV-Hop algorithm.

Table 3. IDV [6] Vs Modified DV-Hop algorithm

\begin{tabular}{|c|c|c|c|}
\hline Proposer & Algorithm & $\begin{array}{c}\text { Localization error }(\text { Area }= \\
100 \times 100 \mathrm{~m}^{2}, \text { Total nodes }= \\
90, \text { Anchor nodes }=60, \\
\text { Range }=15 \mathrm{~m})\end{array}$ & $\begin{array}{c}\text { Localization error }(\text { Area }= \\
100 \times 100 \mathrm{~m}^{2}, \text { Total nodes }=90, \\
\text { Anchor nodes }=60, \text { Range }= \\
20 \mathrm{~m})\end{array}$ \\
\hline $\begin{array}{l}\text { J. Zhang et. al } \\
\text { Glory Pachnanda }\end{array}$ & $\begin{array}{c}\text { IDV } \\
\text { Modified DV-Hop }\end{array}$ & $\begin{array}{c}4.9 \\
1.8699\end{array}$ & $\begin{array}{c}5 \\
1.4322\end{array}$ \\
\hline
\end{tabular}

Table 4. DVI [7] and Modified DV-Hop algorithm

\begin{tabular}{|c|c|c|c|}
\hline Proposer & Algorithm & $\begin{array}{c}\text { Localization error }(\text { Area }= \\
100 \times 100 \mathrm{~m}^{2}, \text { Total nodes }= \\
100, \text { Anchor nodes }=30, \\
\text { Ranqe }=10 \mathrm{~m})\end{array}$ & $\begin{array}{c}\text { Localization error (Area }= \\
100 \times 100 \mathrm{~m}^{2}, \text { Total nodes }=100, \\
\text { Anchor nodes }=30, \text { Range }= \\
40 \mathrm{~m})\end{array}$ \\
\hline $\begin{array}{c}\text { C.Cai et. al } \\
\text { Glory Pachnanda }\end{array}$ & $\begin{array}{c}\text { DVI } \\
\text { Modified DV-Hop }\end{array}$ & $\begin{array}{c}25 \\
8.9895\end{array}$ & $\begin{array}{c}10 \\
3.2494\end{array}$ \\
\hline
\end{tabular}

Table 5. IDV [8] Vs Modified DV-Hop algorithm

\begin{tabular}{|c|c|c|c|}
\hline Proposer & Algorithm & $\begin{array}{c}\text { Localization error }(\text { Area }= \\
50 \times 50 \mathrm{~m}^{2}, \text { Total nodes }=100, \\
\text { Anchor nodes }=30, \text { Range }= \\
10 \mathrm{~m})\end{array}$ & $\begin{array}{c}\text { Localization error }(\text { Area }= \\
50 \times 50 \mathrm{~m}^{2}, \text { Total nodes }=80, \\
\text { Anchor nodes }=30, \text { Range }= \\
10 \mathrm{~m})\end{array}$ \\
\hline $\begin{array}{c}\text { H. Chen et. al } \\
\text { Glory Pachnanda }\end{array}$ & $\begin{array}{c}\text { IDV } \\
\text { Modified DV-Hop }\end{array}$ & $\begin{array}{c}42 \\
7.2888 \\
\end{array}$ & $\begin{array}{c}45 \\
4.7160 \\
\end{array}$ \\
\hline
\end{tabular}

\section{Conclusion}

For the disadvantage of DV-Hop, this paper proposes some improvements over DVHop in order to reduce error. This paper describes the improved scheme in detail. The simulation which is done by deploying nodes in certain area proved that the more anchor nodes considered for the calculation of average hop distance, the lower error is. Modified DV-Hop localization algorithm to improve the poor locating performance of DV-Hop algorithm, mainly focusing on two aspects: firstly, the average one hop distance error of anchor nodes was modified. Secondly, the average hop size is modified and last formula to calculate localization error and accuracy is modified. Simulation result shows that our proposed algorithm can improve location accuracy.

\section{References}

[1] U Aeron and H Kumar. "Coverage Analysis of Various Wireless Sensor Network Deployment Strategies". International Journal of Modern Engineering Research. 2013; 3: 955-961.

[2] J Wang, RK Ghosh and SK Das. "A Survey on Sensor Localization". Journal of Control Theory Application. 2010.

[3] M Vojdani and M Dehghan. "Localization in Anchor Less Wireless Sensor Network". International Proceedings of Computer Science and Information Technology. 2011; 2. 
[4] D Niculesu and B Nath. "Position and Orientation in Ad Hoc Networks". Elsevier Ad Hoc Networks. 2004: 133-151.

[5] H Safa and F Yassine. "Localization in Large Scale Wireless Sensor Networks". 19 ${ }^{\text {th }}$ International Conference on Telecommunications (ICT 2012). 2012.

[6] J Zhang, W Li, D Cui, X Sun and F Zhou. "Study on Improved DV-Hop Node Localization Algorithm in Wireless Sensor Network". $5^{\text {th }}$ IEEE Conference on Idustrial Electronics and Applicationa. 2010: 18551858.

[7] C Cai and L Yuan. "DV-Hop Localization Algorithm Improvement of Wireless Sensor Networks". Journal of Theoretical and Applied Information Technology. 2010; 48: 1546-1551.

[8] H Chen, K Sezaki, P Deng and HC So. "An Improved DV-Hop Localization Algorithm For Wireless Sensor Networks". IEEE Conference on Communications, Networking and Mobile Computing. 2008: 1-4. 